\title{
Prostatic Acid Phosphatase
}

National Cancer Institute

\section{Source}

National Cancer Institute. Prostatic Acid Phosphatase. NCI Thesaurus. Code C18015.

Prostatic acid phosphatase (386 aa, $\sim 45 \mathrm{kDa}$ ) is encoded by the human ACPP gene. This protein is involved in the dephosphorylation of both lipids and tyrosine and has ecto-5'nucleotidase activity. 